\title{
Enhancement of Mechanical Integrity of Advanced Composites using PMAA- Electropolymerised CF Fabrics
}

\author{
Dionysios A. Semitekolos ${ }^{1}$, Panagiotis Goulis ${ }^{1}$, Despoina I. Batsouli ${ }^{1}$, Elias $\mathrm{P}$. \\ Koumoulos $^{1}$, Loukas Zoumpoulakis ${ }^{1}$, and Costas A. Charitidis ${ }^{1, *}$ \\ ${ }^{1}$ National Technical University of Athens, Department of Chemical Engineering, RNANO Lab - \\ Research Unit of Advanced, Composite, Nanomaterials and Nanotechnology, 9 Heroon Polytechneiou \\ st., Zografos Athens, GR-15773
}

\begin{abstract}
The aim of the present study is the development of new composite materials that show improved mechanical and structural integrity. In order to accomplish this goal, a novel functionalization method of the carbon fibers for the reinforcement of the composites surface was investigated. Through the electrografting of methacrylic acid onto the surface of the carbon fiber, this treatment aims to selectively modify the surface of the carbon fabrics, in order to create active groups that can chemically react with the epoxy resin, under heat and pressure. By this way, better adhesion as mechanical interlocking between the resin and the reinforcement can be achieved. The surface treatment was examined qualitatively by means of Infrared spectroscopy, Scanning Electron Microscopy and Raman spectroscopy. The carbon fiber reinforced polymers were manufactured via the hot-press technique and they were subsequently submitted to flexural, shear and nanoindentation test. Finally, the internal structural integrity was tested through micro-Computing Tomography.
\end{abstract}

\section{Introduction}

Carbon fiber (CF) and their use in carbon fiber reinforced polymer composites(CFRPs) have been widely used as structural and functional materials in a variety of applications, from automotive industry and aerospace applications to sports and leisure, due to their astonishing mechanical and physical properties, such as high strength, high modulus, high temperature and fatigue resistance, electrical conductivity and light weight [1,2]. Fiber reinforced polymers are extensively used as reinforcing materials of structures in applications where conventional strengthening techniques may be dubious. CFRPs are regularly made of 55$65 \%$ fibers in epoxy matrices, or fabrics made of unidirectional or bidirectional fibers. However, current fiber reinforced materials have been limited in high performance applications due to poor interfacial fiber/matrix strength [3]. A weak fiber-resin bond leads to poor mechanical properties, such as low interlaminar shear strength (ILSS), which is attributed to a lack of bonding between the resin matrix and fiber filaments. This problem can be overcome by surface treatment of the fiber; however, if the bond is rather strong, the composite becomes brittle and weak. High modulus fibers will require maximum treatment in order to give acceptable properties [4]. A proper engineered interface is crucial to assure the required load transfer from matrix to reinforcements; this will facilitate the relief of internal stress concentrations while improving mechanical integrity and environmental stability of composites efficiently [5]. 
The objective of the present study is the development of new composite materials that show improved mechanical and structural integrity. To accomplish this goal, a novel functionalization method of the carbon fibers surface was investigated, which were used for the reinforcement of the composites. The treatment aims to selectively modify the surface of the CF fabrics through electropolymerization, in order to create active groups that can chemically react with the epoxy resin, under heat and pressure. By this way, better adhesion as mechanical interlocking between the resin and the reinforcement can be achieved, which influences the mechanical integrity of the composite [6].

By electro-polymerizing, poly methacrylic acid (PMAA) on the CF fabric, the formation of hydrogen bonding between carboxyl groups of PMAA and hydroxyl groups of the epoxy resin is promoted, thus creating an interlayer that enhances the fiber-matrix interfacial adhesion without rendering the material brittle via strong chemical bonding [7].

\section{Experimental}

\subsection{Materials and Methods}

A three-part resin system was purchased from HUNTSMAN industries in USA, while the carbon fiber fabric from HEXCEL industries Inc. also in USA. 2,2'-Diaminodiethylamine (HY 943, Sigma-Aldrich) and N, N-Methylenebis (acrylamide) (Acros Organics) were used without further purification. Methacrylic acid (Acros Organics), was double distilled under reduced pressure prior to use. The hydraulic Hot-Press is a Carver, Model $3856 \mathrm{CE}$.

\subsection{Characterization Methods}

Fourier-transform infrared spectroscopy (FTIR) measurements were conducted using a JASCO FT IR 6600 series equipped with an ATR germanium crystal. The surface morphology was estimated via scanning electron microscopy (SEM) using a PHILIPS Quanta Inspect (FEI Company) microscope with W (tungsten) filament $25 \mathrm{KV}$ equipped with EDAX GENESIS (AMETEX PROCESS \& ANALYTICAL INSTRUMENTS). Raman measurements were performed using a Renishaw inVia Micro-Raman spectrometer working in backscattering configuration and equipped with a near-infrared diode laser emitting at 785 $\mathrm{nm}$. The spectra were recorded by focusing the laser beam on the sample surface and adjusting the light power so that $1 \mathrm{~mW}$ is provided for a spot of about $1 \mu \mathrm{m}$ diameter, whereas, for the fitting of the Raman signal, MatLab software was used.

The flexural and shear tests on the produced CFRPs were studied using a compression force dynamometer (Tiedemann Instruments). Nanoindentation testing was performed with Hysitron TriboLab ${ }^{\circledR}$ Nanomechanical Test Instrument, which allows the application of loads from $1 \mathrm{~N}$ to $10 \mathrm{mN}$ and records the displacement as a function of applied loads with a high load resolution $(1 \mathrm{nN})$ and a high displacement resolution $(0.04 \mathrm{~nm})$. The TriboLab® employed in this study is equipped with a Scanning Probe Microscope (SPM), in which a sharp probe tip moves in a raster scan pattern across a sample surface using a three-axis piezo positioner.

The internal structural information of the samples was observed and collected by the compact desk-top Bruker micro-CT, 3D X-ray scan system, SkyScan 1272. The system consists of a microfocus sealed X-ray source which operates at $20-100 \mathrm{kV}$ and $10 \mathrm{~W}(<5 \mathrm{um}$ spot size@4W), an X-ray detector with a maximum resolution of $11 \mathrm{Mp}(4032 \times 2688$ pixels) and a 14bit cooled CCD fiber optically coupled to scintillator.

\subsection{Surface Treatment and Composite Manufacturing}

The surface treatment of the CF fabrics comes in two stages in order to be successful. First of all, each fabric had to be electrochemically treated to enhance the roughness of its surface and to bind oxygen containing groups [8]. To accomplish this, each layer of carbon fabric 
has undergone through cyclic voltammetry test with multiple sweeps in the region of $-2 \mathrm{~V}$ to $2 \mathrm{~V}$, scan rate $100 \mathrm{mV} / \mathrm{s}$ in a sulfuric acid solution of $5 \% \mathrm{w} / \mathrm{w}$.

The second stage includes the electro-polymerization of acrylic acid onto the surface of the CF fabric. The experimental set up and condition have been previously described [7]. For this purpose, a polyacetal cell was used with a volume of $500 \mathrm{ml}$. The CF fabric was used as the working electrode, two stainless steel plates were used as counter electrodes and a saturated silver chloride electrode as reference electrode. The electro polymerization, took place under potentiostatic conditions in an aqueous solution containing $0.3 \mathrm{M}$ acrylic acid, $0.4 \mathrm{M} \mathrm{ZnCl}$ as the supporting electrolyte and $10 \mathrm{mM} \mathrm{N}$, N-Methylene-bis(acrylamide) which acted as a crosslinker for the grafted polymer.

To achieve the required thickness of the composite specimens $(3 \mathrm{~mm}), 10$ layers of G1157 (pristine and surface treated) were placed via hand lay-up method in a matched die mold that was already covered with a thin film of release agent (wax).

For the composite manufacturing process, 8 layers of G1157 (pristine and treated) were placed via hand lay-up method in a matched die mold and afterwards in a hot press (Carver, Model $3856 \mathrm{CE}$ ). The curing took place at $80^{\circ} \mathrm{C}$ for 4 hours at a pressure of $17.2 \mathrm{MPa}$, while the post-curing at $120{ }^{\circ} \mathrm{C}$ for 4 hours. The composites were subsequently cooled in room temperature in order to be able to demold them without been distorted.

\section{Results and Discussion}

\subsection{Fourier-transform infrared spectroscopy}

Figure 1 demonstrates the FT-IR spectra of the pristine and electropolymerized G1157 CF fabric. The pristine fabric exhibits a number of peaks in the mid infrared area. After comparing these results with the mid-IR spectrum of epoxy resins, which are found in the existing literature [9], it was observed that the resulting peaks were quasi identical, thus concluding that the pristine fabric contains epoxy sizing on its outer surface. More specifically, in Figure 1a, the peaks located at $1509 \mathrm{~cm}^{-1}$ and $831 \mathrm{~cm}^{-1}$ can be attributed to C$\mathrm{C}$ single bond stretch vibration of the aromatic ring and the $\mathrm{C}-\mathrm{O}-\mathrm{C}$ stretch vibration of the oxirane group respectively, whereas the peaks found at $1036 \mathrm{~cm}^{-1}$ and $1234 \mathrm{~cm}^{-1}$ can be attributed to the stretch vibrations of C-O-C and C-O of ethers respectively. In Figure $1 \mathrm{~b}$, which represents the PMAA electropolymerized fabric, the spectrum is identical with the pristine with the addition of two peaks located at $1699 \mathrm{~cm}^{-1}$ and $2934 \mathrm{~cm}^{-1}$ which are attributed to the stretch vibrations of $\mathrm{C}=\mathrm{O}$ and $\mathrm{O}-\mathrm{H}$ of the carboxylic acid group respectively, thus indicating the presence of poly methacrylic acid on to the fibers surface.

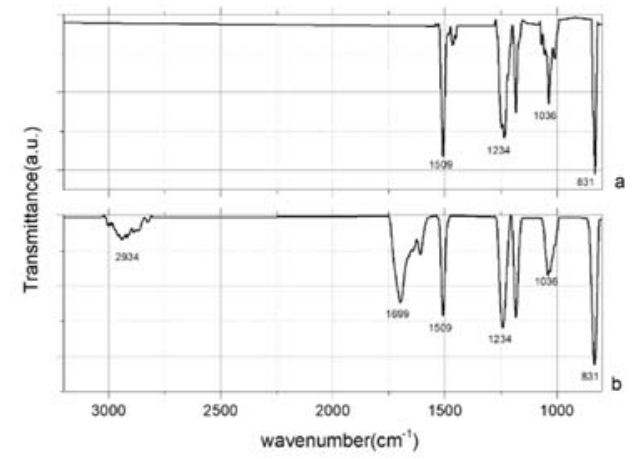

Figure 1. FT-IR spectra of (a) pristine fabric and (b) electropolymerized fabric 


\subsection{Scanning electron microscopy}

The morphological characterization of the modified fibers is represented in Figure 2. Figure $2 \mathrm{a}$ depicts the pristine fiber, prior to any modifications. It can be observed that each monofilament of the pristine fiber exhibits a smooth, striped surface. Figure $2 b$ depicts the fibers after the pre-treatment via cyclic voltammetry. It can be observed that the fiber's surface roughness has increased significantly since the smooth surface of the pristine fiber has been deformed due to the controlled exfoliation phenomenon of the oxidation and reduction cycles. The morphology of the electropolymerized CF fabrics is presented in Figures $2 \mathrm{c}$ and $2 \mathrm{~d}$. The poly methacrylic acid (PMAA) coating can be observed clearly in Figure $2 \mathrm{c}$ as the glossy surface deposited on to the surface of the monofilament, whereas in Figure 2d PMAA coating can be identified as the rigid deposition onto the surface of the monofilaments. As it can be observed by Figures $2 \mathrm{c}$ and $2 \mathrm{~d}$ the electro-polymerization process has produced a homogeneous coating in the outer monofilaments of each bundle, however, certain monofilaments are partially coated. This can, presumably, be attributed to the fact that the electrochemical pre-treatment, as well as the polymer deposition occurs predominantly in the outer monofilaments of each carbon fiber bundle.

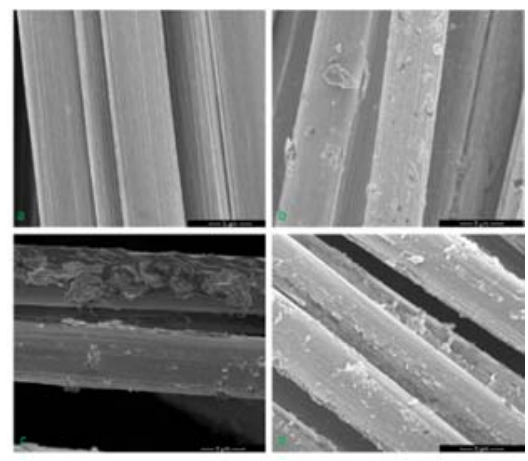

Figure 2. SEM images of (a) pristine fabric, (b) pre-treated fabric through cyclic voltammetry and (c, d) electropolymerized fabric

\subsection{Raman spectroscopy}

Figure 3 illustrates the spectra of the pristine CF fabric (G1157) and the electropolymerized fabric (G1157_PMAA). The peaks observed in both spectra at approximately $1330 \mathrm{~cm}^{-1}$ and $1597 \mathrm{~cm}^{-1}$ are corresponding to the $\mathrm{D}$ and $\mathrm{G}$ peak, respectively. The $\mathrm{D}$ peak is activated by the defects or disorders of the graphitic lattices whereas the $G$ peak is corresponding to the stretching vibrations of the graphitic structures due to the $\mathrm{sp}^{2}$ phase [10-13]. The $\mathrm{I}_{\mathrm{d}} / \mathrm{I}_{\mathrm{g}}$ ratio is a factor that correlates the intensity of the aforementioned peaks and gives information regarding the fiber microstructure heterogeneity and therefore can be an indication of surface defects [14]. The black curves shown on Figure 3 are the spectra of the pristine fabric whereas the colored curves represent the fitting of each peak in order to calculate the $I_{d} / I_{g}$ ratio for each spectrum. The $I_{d} / I_{g}$ ratio was found 1,45 and 2,40 for the pristine and modified CF fabric respectively. Evidently, both fabrics exhibit increased defects, the pristine fabric due to the commercial epoxy sizing as it was shown in the IR analysis and the modified fabric due to the electro polymerization surface modification. The electro polymerization of poly methacrylic acid introduces additional functional groups on to the fibers surface which can deteriorate the graphitic structures and thus lead to a further increase of the $I_{d} / I_{g}[15-16]$. 


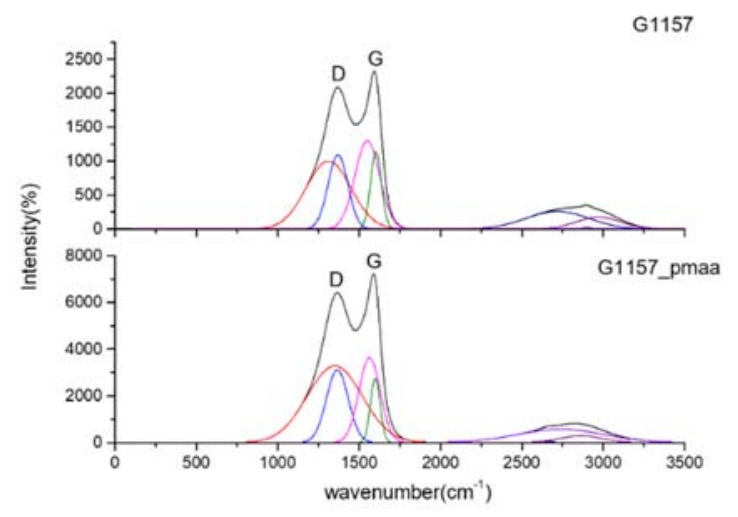

Figure 3. Raman Spectra for pristine (top) and electropolymerized (bottom) fabric

\subsection{Mechanical Performance}

\subsubsection{Flexural Strength Test}

To evaluate the effect on the mechanical performance of composites materials of the electropolymerization, mechanical tests have been performed. The composites were manufactured according to ASTM D790 for the flexural strength test. 8 specimens were tested.

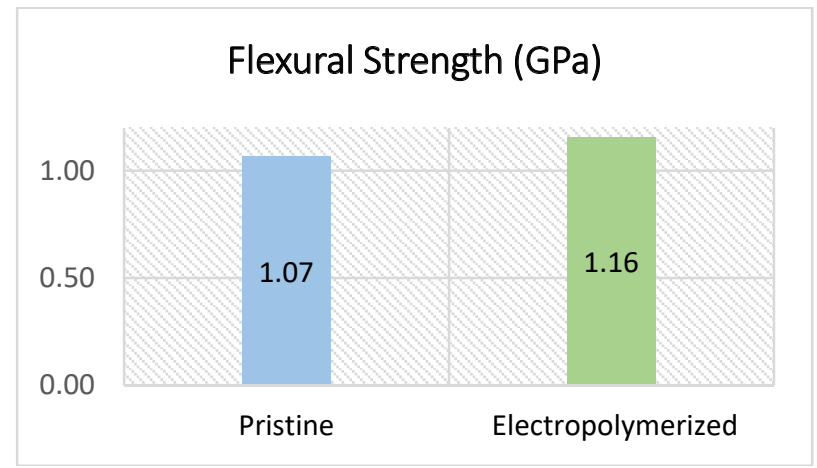

Figure 4. Flexural Strength of composite materials

The composites with pristine CF fabric exhibited a flexural strength of $1.07 \mathrm{GPa}$ whereas the electropolymerized specimens revealed a flexural strength of $1.16 \mathrm{GPa}$. From Figure 4, it is safe to conclude that electropolymerization on the carbon fiber surface can increase the flexural strength of the composite material for $8.67 \%$.

\subsubsection{Interlaminar Shear Strength Test (ILSS)}

For the ILSS test, 8 specimens were manufactured and tested according to ASTM D2344. The composites with pristine CF fabric showed a shear strength of $61,78 \mathrm{MPa}$ while the electropolymerized samples revealed a shear strength of $65,22 \mathrm{MPa}$ (Figure 5). It can be concluded that electrochemical treatment and electropolymerization on the carbon fiber surface can increase the ILSS of the composite material for $5.57 \%$. 


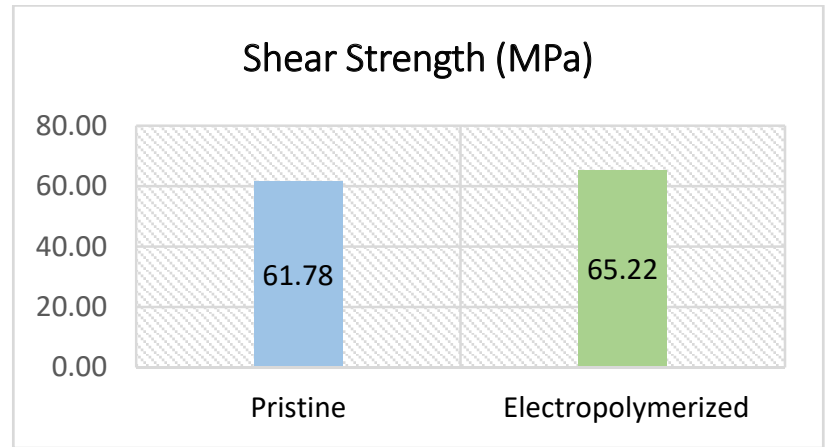

Figure 5. Shear Strength of composite materials

\subsubsection{Nanoindentation Test}

The nanoindentation mapping test was performed on the surface of the composite material and on the cross-section (displacement of $200 \mathrm{~nm}$ ). The probe penetrated in 64 different spots creating a $5 \times 5 \mu \mathrm{m}^{2}$ grid array. On the following figures we can observe the various values of the contact pressure $(\mathrm{GPa})$ as resistance to applied load.
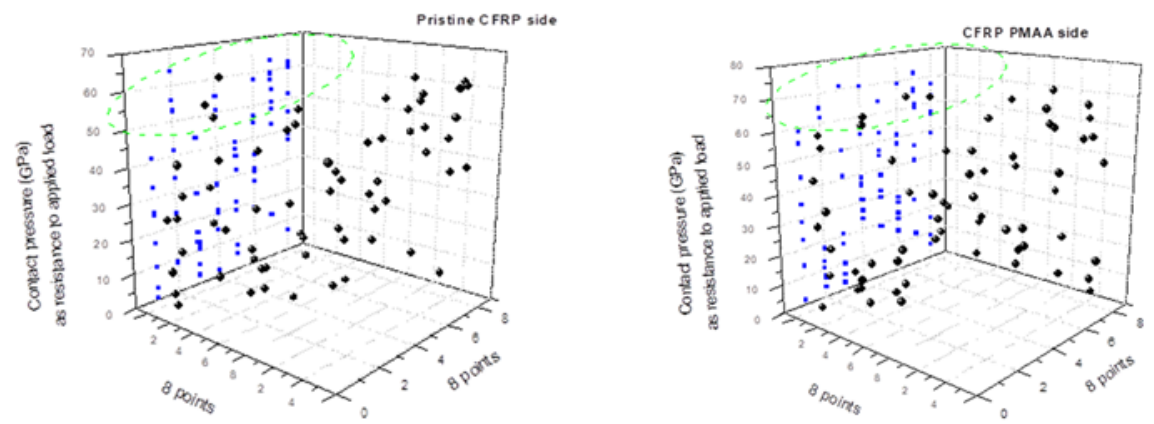

Figure 6. 3D figure of nanoindentation on the composite surface (a) pristine and (b) electropolymerized

From Figure 6, it is observed that in the composite with the electropolymerized fabric the Contact Pressure is higher than in the composite with the pristine fabric.
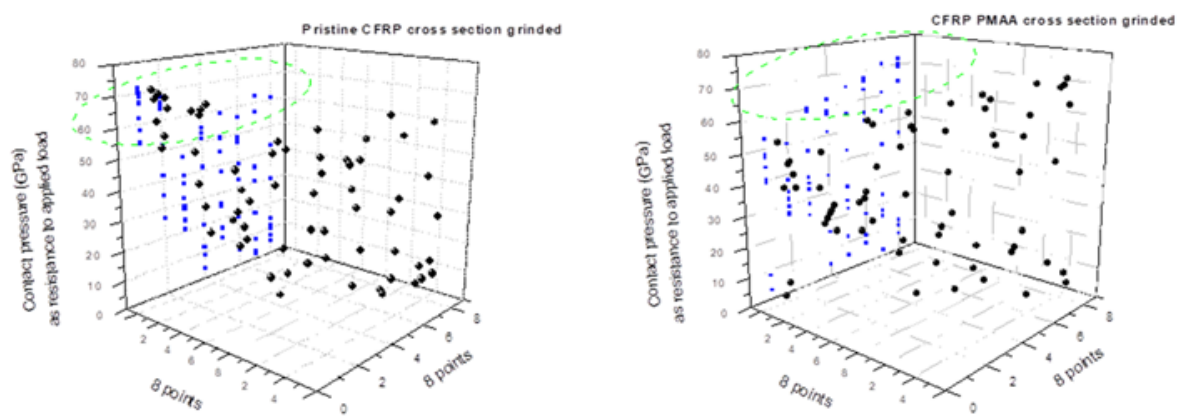

Figure 7. 3D figure of nanoindentation on the cross-section of composite (a) pristine and (b) electropolymerized

In this case, where the cross-section of the composites has been penetrated, the Contact Pressure values of composites with pristine fabric are close to those of the electropolymerized fabric (Figure 7). 


\subsection{Micro-CT X-Ray Tomography}

Regarding the composite fiber samples, it may be mentioned that the presence of PMAA had a substantial impact to the composite properties, as it is evidenced via Micro-CT analysis. PMAA fiber composites demonstrate increased structure thickness. This phenomenon is also ascribed to the higher value of the structure separation. Moreover, the total porosity is also reduced, a fact that shows that PMAA fibers assisted to reduce the inner pores of the material, via their more complex structure. The results of this comparison are presented in Table 3 and the arrangement of the fibers into the epoxy resin matrix is demonstrated in Figure 8.

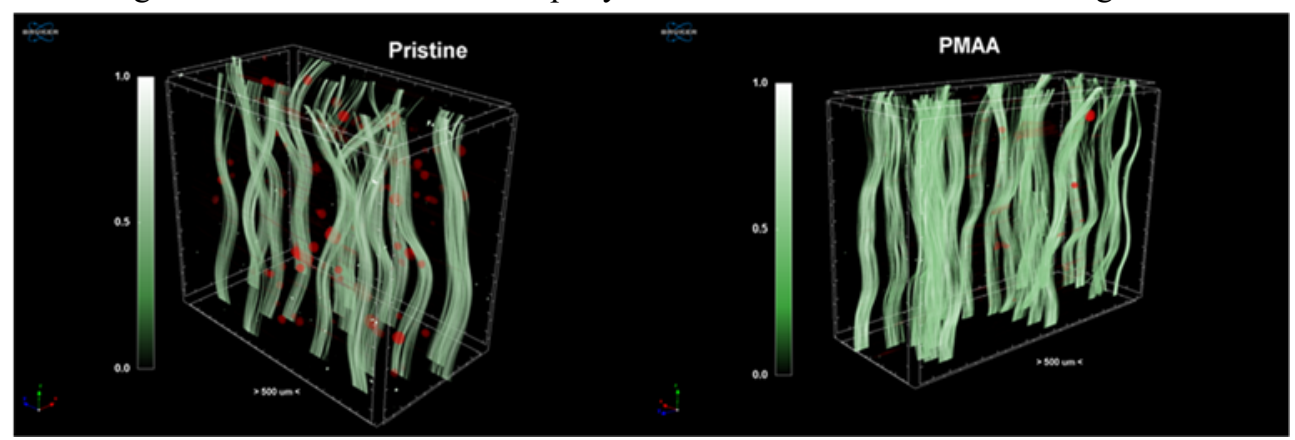

Figure 8. Arrangement of fibers into the epoxy resin matrix via Micro-CT visualization (red = pores).

Table 1. Micro-CT arithmetical results for the fiber epoxy composites

\begin{tabular}{ccc}
\hline Parameters & Pristine & PMAA \\
\hline Object Surface Density $\left(\mathrm{mm}^{-1}\right)$ & 3.974 & 1.793 \\
Structure Thickness $(\mathrm{mm})$ & 0.282 & 0.553 \\
Structure Separation $(\mathrm{mm})$ & 0.051 & 0.227 \\
Structure Linear Density $\left(\mathrm{mm}^{-1}\right)$ & 3.419 & 1.752 \\
Degree of Anisotropy & 3.817 & 2.262 \\
Fractal Dimension & 2.558 & 2.279 \\
Total Porosity $(\%)$ & 3.677 & 3.129 \\
\hline
\end{tabular}

\section{Conclusions}

In this study, a new approach concerning the efficiency of the electro-polymerization of poly methacrylic acid (PMAA) on commercially available carbon fiber fabrics was conducted, in terms of mechanical and structural integrity of the resulting composite specimens, produced by the hot-press technique.

The deposited polymer was characterized qualitatively via IR and Raman Spectroscopy, confirming the polymer deposition on the CF surface, whereas the morphology was investigated via SEM microscopy. The macro scale mechanical testing showed promising results, as both flexural and shear strength were increased by $8.67 \%$ and $5.57 \%$ respectively, as well as an improvement to the Contact Pressure in nanoscale testing can imply that electropolymerization is an effective technique. Micro Tomography confirmed that through this enhancement in adhesion the porosity of the composite material is reduced. Evidently, the electro-polymerization of PMAA on to carbon fiber fabrics can be a powerful tool to enhance the fiber-matrix interfacial adhesion, an environmentally friendly procedure since it takes place in aqueous media. 
This work was supported by the EU H2020 Project "Modified Cost Effective Fibre Based Structures with Improved Multi-Functionality And Performance" (MODCOMP) under Grant Agreement no. 685844 .

\section{References}

1. Morgan P. CRC Press TFG, 951-1042 (2005)

2. Chen Hui, et al., Appl. Surf. Sc. 441, 538-545 (2018)

3. C. González, et al., Prog. Mat. Sci. 89, 194-251 (2017)

4. V. Oliveira, et al., Opt. and Las. in Eng. 94, 37-43(2017)

5. Paul R., Dai L., Comp. Int., 25, 539-605 (2018)

6. Harris B, et. al., Journ. of Mat. Sci., 28, 3353-66. (1993)

7. Panayiotis Kainourgios, et Al., Appli. Surf. Sci. 416, 593-604 (2017)

8. Georgiou P, Walton J, Simitzis J., El. Act., 55, 1207-1216 (2010)

9. María González, et. al., Mat. Sci., Eng. and Techn., Review, 4524 (2012)

10. X. Sui, Z. Xu, C. Hu, L. Chen, L. Liu, L. Kuang, M. Ma, L. Zhao, J. Li, H. Deng, Compos. Sci. Technol. 130, 46-52 (2016)

11. G. Washer, F. Blum, Res. Lett. Mater. Sci. 2008 1-3 (2008)

12. G.-z. Ren, C.-j. Chen, L.-h. Deng, H.-y. Quan, Y.-g. Lu, Q.-1. Wu, New Carb. Mat. 30, 476-480 (2015)

13. S. Wu, Y. Liu, Y. Ge, L. Ran, K. Peng, M. Yi, Comp. Part A: Appl. Sci. Manuf. 90, 480-488 (2016)

14. P. Musiol, P. Szatkowski, M. Gubernat, A. Weselucha-Birczynska, S. Blazewicz, Ceram. Int. 42, 11603-11610 (2016)

15. M. Zhao, L. Meng, L. Ma, G. Wu, Y. Wang, F. Xie, Y. Huang, RSC Adv. 6, 2965429662 (2016)

16. L. Mao, Y. Wang, Z. Zang, S. Zhu, H. Zhang, H. Zhou, J. Appl. Polym. Sci.131, 40274-40282 (2014) 